\title{
Competition between Order and Phase Separation in Au-Ni
}

\author{
H. Reichert, ${ }^{1}$ A. Schöps, ${ }^{1}$ I. B. Ramsteiner, ${ }^{1}$ V. N. Bugaev, ${ }^{1}$ O. Shchyglo, ${ }^{1}$ A. Udyansky, ${ }^{1}$ H. Dosch,${ }^{1,2}$ M. Asta, ${ }^{3}$ \\ R. Drautz, ${ }^{4}$ and V. Honkimäki ${ }^{5}$ \\ ${ }^{1}$ Max-Planck-Institut für Metallforschung, Heisenbergstrasse 3, D-70569 Stuttgart, Germany \\ ${ }^{2}$ Universität Stuttgart, Institut für Theoretische und Angewandte Physik, D-70569 Stuttgart, Germany \\ ${ }^{3}$ Northwestern University, Evanston, Illinois 60208, USA \\ ${ }^{4}$ Department of Materials, University of Oxford, Oxford OX1 3PH, United Kingdom \\ ${ }^{5}$ European Synchrotron Radiation Facility, F-38043 Grenoble, France
}

(Received 3 August 2005; published 2 December 2005)

\begin{abstract}
We have measured and theoretically analyzed the diffuse scattering in the binary alloy system Au-Ni, which has been proposed as a testing ground for theories of alloy phase stability. We found strong evidence that in the alloys $\mathrm{Au}_{3} \mathrm{Ni}$ and $\mathrm{Au}_{3} \mathrm{Ni}_{2}$, fluctuations of both ordering- and clustering-type are competing with each other. Our results resolve a long-standing controversy on the balance of relaxation and mixing energies in this alloy system and explain recent findings of ordering in thin Au-Ni films.
\end{abstract}

DOI: 10.1103/PhysRevLett.95.235703

One future vision for advanced multicomponent materials is that their structural properties can be modeled accurately by $a b$ initio theories, allowing the controlled synthesis of material structures with tailored functions. The challenge is to understand the intrinsic interactions between the constituent atoms with sufficient precision in order to predict the structure and the phase behavior of the materials. The simplest materials systems which can be used as test beds for our current microscopic understanding of multicomponent materials are binary alloys $A_{1-c} B_{c}$, grown as single crystals from a solution of atoms $A$ and $B$ (with $B$-concentration $c$ ).

Binary alloys are generally classified into two generic types of ordering or phase separating character. The phase behavior of a binary $A-B$ system is determined by a balance between the interactions $V_{A A}, V_{B B}$, and $V_{A B}$ which are typically of the order of $1 \mathrm{eV}$. The resulting effective pair interaction $V=V_{A A}+V_{B B}-2 V_{A B}$, on the other hand, is typically of the order of several $10 \mathrm{meV}$, and, thus, competing with the thermal excitations in the system. In turn, only the accurate determination of these interactions by experiment and first-principles calculations allows predictions to be performed on alloy phase stability for a given system $[1,2]$.

In many cases the phase behavior of a given binary alloy system can be deduced from the short-range order fluctuation spectrum in the high temperature disordered phase [3]. Ordering systems show fluctuations with a dominant wave vector $\vec{k} \neq \overrightarrow{0}$ [see Fig. 1(a)], while fluctuations in phase separating systems are generally characterized by a dominant wave vector $\vec{k}=\overrightarrow{0}$. The two cases can easily be distinguished via the diffuse $\mathrm{x}$-ray or neutron scattering caused by the fluctuations in the system [4].

However, there are many systems where such a simple picture fails. Some alloy systems can indeed form a phase separated ground state while the high temperature fluctuations are of both, ordering- and/or clustering-type. A sys-
PACS numbers: 64.60.Cn, 34.20.Cf, 61.10.-i, 61.66.Dk

tematic analysis of the energies involved reveals that it is the balance of three different types of energies, (a) the formation enthalpy of ordered structures, (b) the mixing enthalpy of the random alloy, and (c) the strain energy from atomic size mismatch which allows the classification of all systems into five alloy types, ranging from strongly ordering to strongly clustering alloys [5].

One of these alloy systems is Au-Ni, which is of particular interest as it developed into a controversial and puzzling case. Figure 1(b) depicts the phase diagram of the Au-Ni system. At high temperatures it shows a homogeneous solid fcc solution with a miscibility gap spanning the entire concentration range. From this simple phase diagram it has been assumed that the disordered high temperature phase should only show fluctuations of the clustering-type. On the other hand, state-of-the-art first-
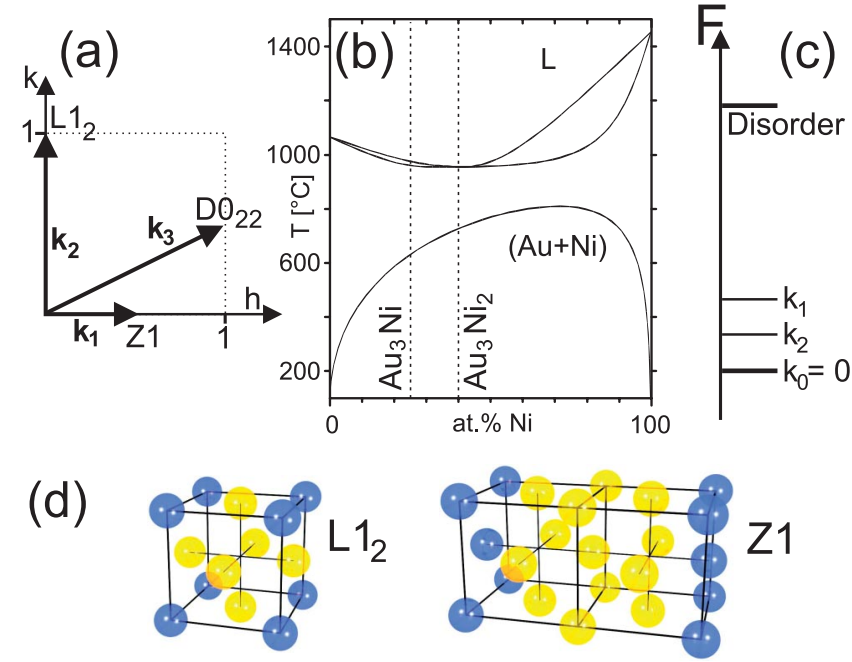

FIG. 1 (color). (a) Ordering wave vectors in the fcc system; (b) phase diagram of the Au-Ni system; (c) competition between several ordered states and phase separation; (d) $L 1_{2}$ (left) and $Z 1$ (right) structure in real space. 
principles calculations by Wolverton et al. display a rich short-range order pattern indicating competing fluctuations of both ordering- and clustering-type in the bulk alloy [6,7]. In turn the short-range order (SRO) diffuse scattering in this system is predicted to be intermediate between that of a strongly ordering alloy and that of a strongly clustering alloy [5].

On the experimental side this has been a rather contradictory case. Early measurements of the diffuse scattering indeed indicated clustering-type fluctuations (see [8] and references therein). This has been challenged in diffuse scattering measurements at $\mathrm{Au}_{3} \mathrm{Ni}_{2}$ by $\mathrm{Wu}$ and Cohen, where some faint diffuse intensity maxima at $\vec{k}=$ $\{0.6,0,0\}$ positions have been observed [9]. This points directly to the existence of ordering fluctuations in this system. More recently, diffuse neutron scattering experiments found again no evidence for ordering fluctuations associated with a wave vector $\vec{k} \neq \overrightarrow{0}[8,10]$.

In this Letter we present new diffuse scattering experiments of very high accuracy and resolution obtained by a novel high energy x-ray diffraction technique [11]. The high quality of the data and a new $k$-space theoretical analysis of the findings allow us to identify unambiguously the various types of fluctuations in the system and their underlying driving forces. This new insight into this system settles the controversy on the topology of the SRO and allows us to explain a recent observation in $\mathrm{Au}-\mathrm{Ni}$ films grown on Au substrates. Upon annealing, these films exhibit long-range ordering in the $Z 1$ structure which appears to be in conflict with the bulk phase diagram [12].

Diffuse scattering measurements give unique experimental access to the Fourier transform of the effective interatomic pair potential $V_{\vec{k}}$ [4]. It is encoded in the diffuse scattering intensity via the Warren-Cowley SRO parameters $\alpha_{\vec{k}}$ in the Krivoglaz-Clapp-Moss equation

$$
\alpha_{\vec{k}} \sim\left[1+\frac{c(1-c)}{k_{B} T} V_{\vec{k}}\right]^{-1},
$$

where $c$ denotes the concentration. It can be seen directly from Eq. (1) that the minima in the effective pair potential correspond to maxima in the diffuse scattering distribution. Recently developed $k$-space models which represent $V_{\vec{k}}$, as the sum of a chemical contribution and a strain-induced contribution, $V_{\vec{k}}=V_{\vec{k}}^{\text {ch }}+V_{\vec{k}}^{\text {si }}$, enable us to identify the origin of the individual components of the fluctuation spectrum for a given system [13]. This is of particular importance for the system Au-Ni as strain-induced contributions to the total effective pair interaction are expected to be large due to the large size mismatch of about $15 \%$ between $\mathrm{Au}$ and $\mathrm{Ni}$.

The measurements have been performed at beam lines ID15A (ESRF) and X17B1 (NSLS, BNL). The energy of the $\mathrm{X}$-ray beam has been varied between 60 and $90 \mathrm{keV}$ in order to tune the multiple scattering from the macroscopically thick sample. The $\mathrm{Au}_{3} \mathrm{Ni}$ and $\mathrm{Au}_{3} \mathrm{Ni}_{2}$ single crystal samples with a (001) surface normal were cut and polished to a thickness of $0.6 \mathrm{~mm}$. During the experiments the samples have been mounted on a boron-nitride heater specially designed for in situ diffuse scattering experiments in transmission geometry. The sample assembly has been mounted in a vacuum chamber in order to reduce air scattering. Figure 2 sketches the experimental setup: The sample is oriented with a high symmetry direction parallel to the high energy x-ray beam. The scattering pattern is then detected in transmission geometry, while the primary $\mathrm{x}$-ray beam is absorbed by a beam stop. Because of the large radius of the Ewald sphere in the high energy x-ray regime, the $2 \mathrm{D}$ detector records extended areas in reciprocal space within very short exposure times and the deviation of the momentum transfer from the corresponding high symmetry plane is small within the first Brillouin zone.

The scattering patterns have been recorded in single exposures within a few seconds from quenched samples as well as from in situ measurements using both an x-ray CCD camera (MARCCD) and an online image plate system (MAR345). Figure 3 shows the diffuse intensity pattern for quenched samples of $\mathrm{Au}_{3} \mathrm{Ni}$ (a) and $\mathrm{Au}_{3} \mathrm{Ni}_{2}$ (b) at an x-ray energy of $62.5 \mathrm{keV}$. The scattering patterns have been corrected for absorption, Compton scattering, and polarization effects. Close to the $\{2,0,0\}$ positions thermal diffuse scattering and so-called Huang diffuse scattering from static lattice distortions are dominant. The diffuse scattering patterns for both alloys are similar with only slight changes in the position of the diffuse scattering maxima. Most interestingly, we find strong diffuse intensity maxima at several positions within the Brillouin zone (marked in white in the upper right part of the patterns). The maxima are produced by fluctuations with dominant wave vectors $\vec{k}_{1}=\{0.5,0,0\}$ and $\vec{k}_{2}=\{1,0,0\}$ [see Fig. 3(d)], revealing fluctuations into $Z 1$ and $L 1_{2}$ type structures, respectively, [see Fig. 1(d) for the corresponding real-space structures]. The diffuse scattering maxima at the $\vec{k}_{2}$ positions are split due to Fermi surface nesting

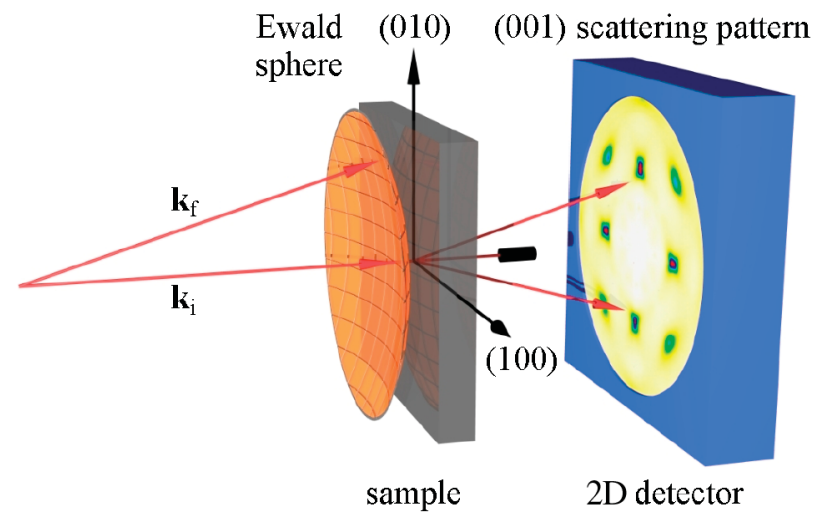

FIG. 2 (color). Sketch of the experimental setup for high energy $x$-ray diffuse scattering in transmission geometry. The Ewald construction shows that for high energy $x$ rays the deviation from a plane is very small in the first Brillouin zone. 

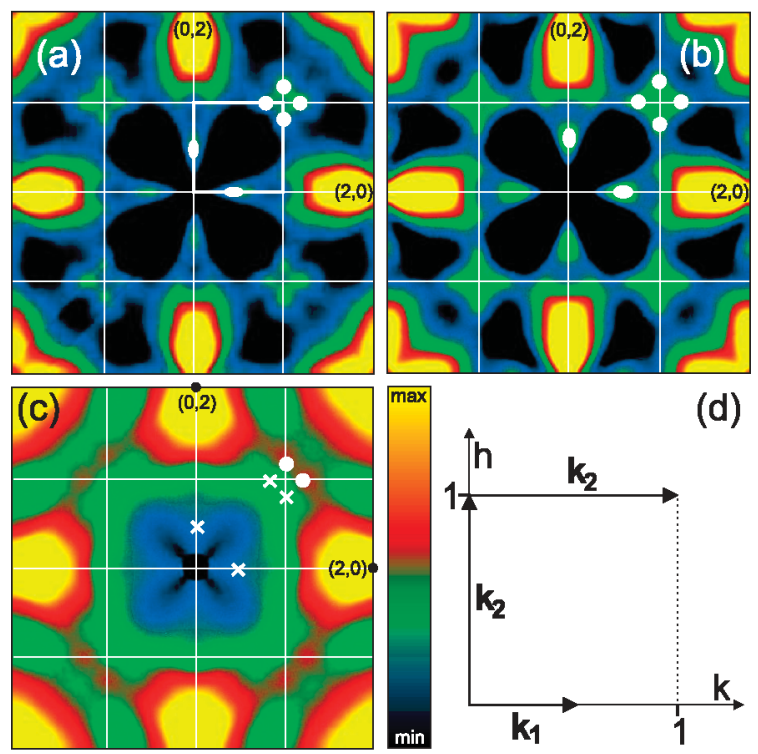

FIG. 3 (color). Diffuse scattering in the (001) plane for (a) $\mathrm{Au}_{3} \mathrm{Ni}$ and (b) $\mathrm{Au}_{3} \mathrm{Ni}_{2}$ measured from quenched samples at room temperature. The positions of the principal diffuse scattering maxima are marked in the upper right part of the first Brillouin zone. (c) Diffuse scattering in the (001) plane for $\mathrm{Au}_{3} \mathrm{Ni}$ measured in situ at $T=700{ }^{\circ} \mathrm{C}$. (d) Principal wave vectors for the fluctuations in $\mathrm{Au}-\mathrm{Ni}$ [bold square in (a)].

effects and asymmetric due to strong lattice distortions (the split peaks around the $\{1,0,0\}$ positions itself are not visible as separate peaks due to additional distortioninduced scattering). Figure 3(c) shows the diffuse scattering distribution for $\mathrm{Au}_{3} \mathrm{Ni}$ kept at $T=700{ }^{\circ} \mathrm{C}$ in the disordered high temperature phase with the expected increase in the thermal diffuse scattering. The stronger pair of diffuse satellites around the $\{1,1,0\}$ positions is still detectable, while the diffuse scattering peaks at the $\vec{k}_{1}=$ $\{0.5,0,0\}$ positions are too weak to be detected.

Our observations give clear-cut experimental evidence for the existence of several different strong order fluctuations in Au-Ni alloys. We observe two order fluctuations associated with the wave vector $\vec{k}_{1}=\{0.5,0,0\}$ and $\vec{k}_{2}=$ $\{1,0,0\}$. In order to shed light onto the nature of the underlying interactions, we have performed first-principles calculations. The formation energies of 22 structures have been obtained from $a b$ initio total energy calculations within the generalized gradient approximation (GGA) for $\mathrm{Au}_{3} \mathrm{Ni}$. The structures have been chosen according to the recently developed topological refinement procedure [14]. For the determination of the chemical contribution $V_{\vec{k}}^{\mathrm{ch}}$, the GGA calculations have been performed for the given set of volume relaxed structures. The long-ranging $V_{\vec{k}}^{\text {si }}$ is obtained from a Kanzaki-Krivoglaz model with only a nearest-neighbor Kanzaki force, $\phi_{1}=-0.55 \mathrm{eV} / \AA$. For the construction of the $k$-space interaction potential we again followed the topological refinement procedure. The results for $V_{\vec{k}}^{\mathrm{ch}}, V_{\vec{k}}^{\mathrm{si}}$, and $V_{\vec{k}}$ for $\mathrm{Au}_{3} \mathrm{Ni}$ are shown in Fig. 4 in

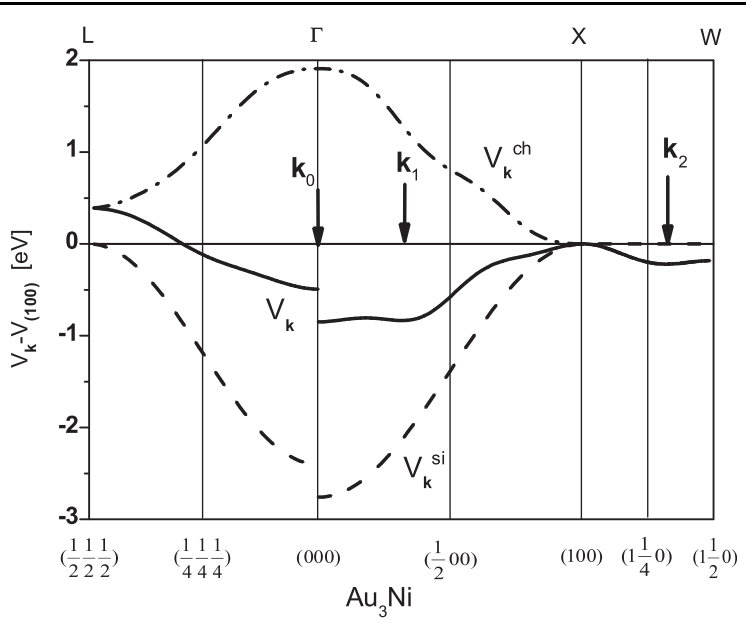

FIG. 4. Fourier components of the chemical and the straininduced part of the atomic interaction, together with the effective interaction potential. The minima in the effective interaction potential are marked by arrows.

a reduced Brillouin zone scheme. $V_{\vec{k}}^{\text {ch }}$ results from a realspace fitting procedure take into account chemical interactions in 10 coordination shells.

The theoretical analysis reveals several remarkable features. It shows first of all that it is not the chemical interaction but rather the strain-induced interaction which is responsible for the phase separation behavior of $\mathrm{Au}-\mathrm{Ni}$ alloys. A similar result has been obtained within the embedded atom method [15]. Second, $V_{\vec{k}}$ exhibits several minima located at or around the principal wave vectors $\vec{k}_{0}=0, \vec{k}_{1}=\{0.5,0,0\}$, and $\vec{k}_{2}=\{1,0,0\}$, in excellent agreement with the experimental findings. Notice in particular, that the concentration fluctuations associated with $\vec{k}_{1}=\{0.5,0,0\}$ are caused by the interplay between the strongly repulsive $V^{\text {ch }}$ and the attractive $V^{\text {si }}$ giving rise to noticeable Z1-type fluctuations. They favor local ordered structures with $3 \mathrm{Au}(100)$ planes followed by $1 \mathrm{Ni}(100)$ plane. As the driving force for this type of fluctuations is strain, one would assume that it is possible to influence their energy by applying external stress. In fact, $Z 1$ structures have been discovered in Au-Ni films grown epitaxially on $\mathrm{Au}(001)$ [12], which are, according to our results, frozen $Z 1$ fluctuations stabilized by epitaxial strain in these Au-Ni nanostructures.

The tendency of the system towards phase separation manifests itself in the diffuse intensity distribution close to the origin of reciprocal space, $k=0$. Figure 5(a) shows a blowup of our experimental data close to the $\Gamma$ point [lower half of Fig. 5(a)] disclosing a rather complex diffuse scattering which is intimately related to the nonanalytic behavior of $V_{\vec{k}}$ at $\vec{k}=\overrightarrow{0}$ (see Fig. 4). This peculiar " $\vec{k}=\overrightarrow{0}$ " phenomenon is caused by the long-ranged part of $V^{\text {si }}$ and emerges unavoidably in systems with a noticeable anisotropic elastic response function [11]. The upper part of Fig. 5(a) shows a simple model calculation including the strain-induced part $V_{\vec{k}}^{\text {si }}$ (taken from Ref. [16]). The com- 

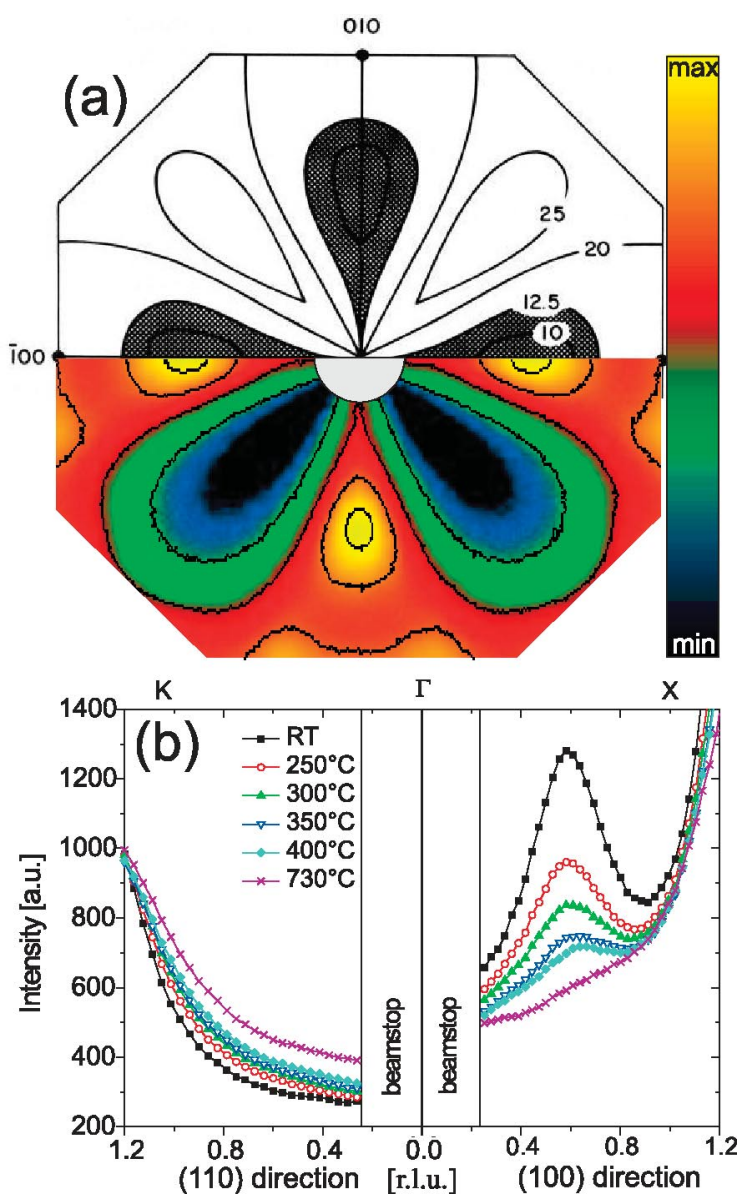

FIG. 5 (color). Diffuse scattering in the (001) plane for $\mathrm{Au}_{3} \mathrm{Ni}_{2}$ in the first Brillouin zone. (a) The upper part displays model calculations taken from Ref. [16], the lower half shows the measured data from quenched samples at room temperature with the central part shadowed by the beam stop. (b) Temperature dependence of the diffuse scattering along $\Gamma-K$ and $\Gamma-X$.

parison reveals complete coincidence between the measured and the calculated pattern with maxima in the diffuse scattering at the locations of the minima in $V_{\vec{k}}^{\text {si }}$. The diffuse intensity maxima within the Brillouin zone clearly indicate the tendency to form ordering fluctuations in the system $\mathrm{Au}-\mathrm{Ni}$, although the ground state of the system corresponds to phase separation. This situation is illustrated in Fig. 1(c), where a phase separated ground state $(\vec{k}=\overrightarrow{0})$ is slightly more favorable in energy than the competing ordered states $\left(\vec{k}_{1}\right.$ and $\left.\vec{k}_{2}\right)$.

The nonanalytic behavior of the diffuse scattering in the vicinity of the origin becomes even more apparent in temperature dependent measurements. For the measurements a $\mathrm{Au}_{3} \mathrm{Ni}_{2}$ crystal has been annealed in the high temperature disordered phase, followed by a quench to a final temperature within the miscibility gap where the diffuse scattering pattern has been recorded. Before the system was able to start decomposition the temperature has been raised again to the high temperature disordered phase. Figure 5(b) shows the diffuse scattering extracted along the lines $\Gamma-K$ and $\Gamma-X$ for a set of temperatures above and below the miscibility gap. It is apparent, that the gap between the measured intensities upon approaching the $\Gamma$ point from two different directions, is increasing with decreasing temperature. The temperature dependence of the diffuse maxima at $\{0.56,0,0\}$ positions demonstrates that entropy finally overcomes the ordering tendencies in the system. Fluctuations into the $Z 1$ type structure gradually disappear upon heating.

In conclusion, we have shown that the system $\mathrm{Au}-\mathrm{Ni}$ exhibits a competition between ordering and phase separation. Fluctuations of both the ordering- and clusteringtype and their driving forces could be identified via the location of their diffuse scattering maxima. The principle features of the diffuse scattering distribution are well reproduced by first-principles calculation of the Au-Ni system [7], while the remaining differences are of a quantitative nature. Our results demonstrate that the topology of the diffuse scattering of the bulk system can be used to understand the phase behavior of the thin film system as well. Systems with competing interactions producing tendencies for both ordering and clustering fluctuations are particularly well suited for a modification of their phase behavior, since the balance between the chemical and strain-induced interactions can be tailored by the epitaxial strain induced by the substrate.

[1] D. de Fontaine, Solid State Phys. 34, 73 (1979).

[2] F. Ducastelle, Order and Phase Stability in Alloys (NorthHolland, Amsterdam, 1991).

[3] A. G. Khachaturyan, Theory of Structural Transformations in Solids (Wiley, New York, 1983).

[4] M.A. Krivoglaz, Diffuse Scattering of X-Rays and Neutrons by Fluctuations (Springer-Verlag, Berlin, 1996).

[5] C. Wolverton, V. Ozolins, and A. Zunger, J. Phys. Condens. Matter 12, 2749 (2000).

[6] C. Wolverton and A. Zunger, Comput. Mater. Sci. 8, 107 (1997).

[7] C. Wolverton, V. Ozolins, and A. Zunger, Phys. Rev. B 57, 4332 (1998).

[8] M. J. Portmann et al., Phys. Rev. B 65, 024110 (2002).

[9] T. B. Wu and J. B. Cohen, Acta Metall. 31, 1929 (1983).

[10] M. J. Portmann et al., Phys. Rev. B 68, 012103 (2003).

[11] H. Reichert et al., Phys. Rev. Lett. 87, 236105 (2001).

[12] C. Dressler et al., Appl. Phys. Lett. 72, 2241 (1998).

[13] V. N. Bugaev et al., Phys. Rev. B 65, 180203 (2002).

[14] O. Shchyglo et al. Phys. Rev. B 72, 140201(R) (2005).

[15] M. Asta and S. M. Foiles, Phys. Rev. B 53, 2389 (1996).

[16] D. de Fontaine and H.E. Cook, in Critical Phenomena in Alloys, Magnets, and Superconductors, edited by E. A. R.E. Mills and R. Jaffe (McGraw-Hill, New York, 1971). 